\title{
The Contribution of Amateur Astronomers to the Study of Variable Stars
}

\author{
Dominique Proust ${ }^{1}$ and Emile Schweitzer ${ }^{2}$ \\ ${ }^{1}$ Département d'Astrophysique Extragalactique et de Cosmologie, \\ Observatoire de Meudon, F-91295 Meudon Principal Cedex, France \\ ${ }^{2}$ Association Française des Observateurs d'Etoiles Variables, \\ F-Strasbourg, France
}

The observation of variable stars is one of the most important and the most fruitful areas of stellar astronomy. The contribution of large numbers of visual observations is a determining factor in drawing up light-curves, the latter being the key to the interpretation of the process of variability within these stars.

It is not easy for a professional astronomer to obtain access to modern telescopes, especially to those of large aperture, and it may even be difficult, given the large number of projects put forward. In any case, these large instruments are often unsuitable for the observation of variable stars. Amateurs, on the other hand, have instruments that have a lower degree of precision, but their greater number and the good organization that exists for the reduction of data obtained, represent trump cards in preparing light-curves.

The amateur thus has a wide gap that can be exploited by using a modestlysized telescope in conjunction with a physiological, rather than physical, detector, the eye, which is capable of making measurements, which are of sufficient accuracy for most types of variation to be scientifically useful.

The development of astronomy from space has also opened up new observational possibilities (with the IUE and IRAS satellites, for example); variable stars radiate in most of the principal regions of the spectrum, so it is possible to make coordinated observations, the visible region being reserved for the amateurs.

Thanks to observations made by the members of the Association Française des Observateurs d'Etoiles Variables (AFOEV) and linked to well-established scientific programmes (Schweitzer and Proust, 1987), numerous results have been obtained in the last few years, over a whole range of types of variation.

\section{Mira-Ceti-type Stars}

The intrinsic properties of these stars (long periods and large-amplitude variations) make them ideal candidates for amateur observation. The light-curves established (Fig. 1) enable the following studies to be carried out:

- Correlations between the magnitude and the variation of other spectral or photometric characteristics (OH, $\mathrm{H}_{2} \mathrm{O}, \mathrm{SiO}$ masers, cf Fillit et al., (1977); study of the radial velocities of the absorption lines). 


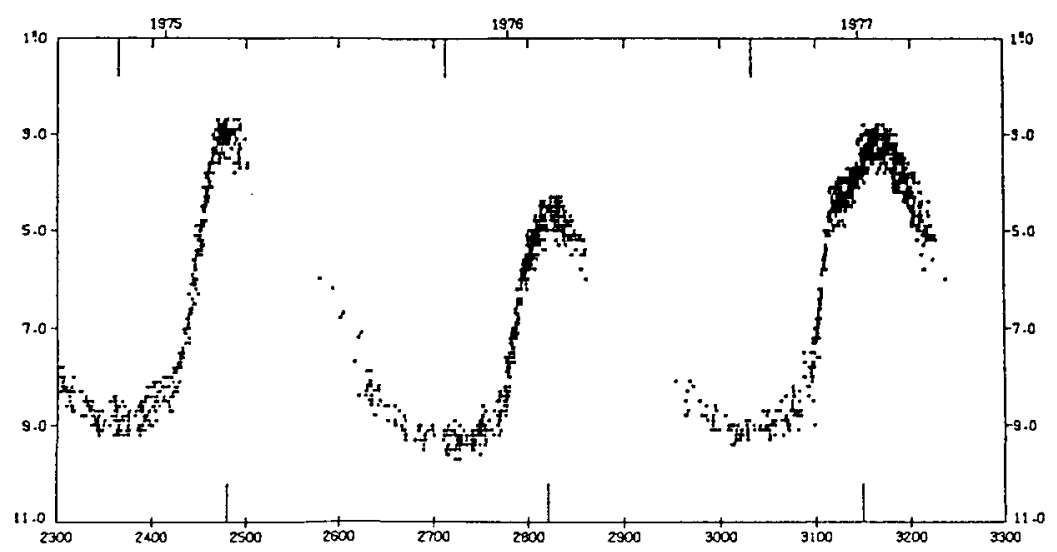

- Use of the parameters of the light-curves, averaged over a large number of cycles (statistical links between these parameters and the final evolution to the white-dwarf, or planetary-nebula stages).

- The physics of Mira stars by studying the cycle-to-cycle variations (stellar structure, dynamical evolution, mass-loss: of Wood, 1982).

- Fourier analysis of the light-curves and establishing ephemerides for solar (Speckle) or space missions (Hipparcos):

$$
m(t)=m_{0}+\sum_{i=1}^{3} A_{i} \cos \left(2 \pi \nu_{i}\left(t-t_{0}+\phi_{i}\right)\right.
$$

where $i=1$ corresponds to the principal mode $\left(\nu_{1}=1 / p\right), p$ being the star's primary period;

$i=2$ is this mode's harmonic $\left(\nu_{2}=2 / p\right)$;

$i=3$ corresponds to the long-term variation that is shown by almost all Miras when their light-curves are analyzed by Fourier methods;

$m_{0}, A_{1}$ and $\nu_{1}$ are taken from the values given in the GCVS (Kukarkin et al.);

$\phi_{1}$ is taken to be equal to $\pi$ and $t_{0}$ to the date of the last maximum observed (Mennesier, 1987).

\section{Other Variables}

The observation of variable stars is not, of course, limited to Miras; the study of stars at the end of their evolution that are found in the centre of planetary nebulae (Acker and Jasniewicz, 1985), is a fundamental tool in understanding the processes that lead to the formation of planetary nebulae. Where novae and supernovae are concerned, amateur astronomers have a vital role, because their light-curves are precious tools in understanding the physics of these objects, as well as the major role of supernovae in the chemical evolution of galaxies, and their enrichment in metals and radioactive elements. The observation of Z-Cam-type stars enables us to refine our models of 
accretion disks that are required to explain certain features of the light-curves in the standstill phase (short duration and fluctuations of minima). The phenomena that occur in symbiotic stars are still too poorly understood for us to explain fully the transfer of material in close systems.

All these examples clearly illustrate the vital role played by variable-star observers. Professionals involved in stellar astronomy more and more frequently request observations. The increasing number of simultaneous observations brings larger and larger forces into play, far transcending national boundaries, in particular by mobilizing amateur and professional observers of variables. Despite this cooperation, a large number of questions about variability remain unanswered or have only partial answers. Amateur astronomers can help to solve these problems.

\section{References}

Acker, A., Jasniewicz, G., 1985: Astron. Astrophys., 143, L1

Fillit, R., Proust, D., Lepine, J.R.D., 1977: Astron. Astrophys., 58, 281

Kukarkin, B.V.: General Catalogue of Variable Stars

Mennessier, M.D.: 1987, INCA report

Schweitzer, E., Proust, D., 1987: L'Astronomie, 101, 303

Wood, P.R., 1982: Pulsation in classical and cataclysmic variable stars, eds. Cox, J.P. and Hansen, C.J. (Joint Institute for Laboratory Astrophysics, Boulder, Colorado) 